\title{
An evolutionarily conserved, alternatively spliced, intron in the p68/DDX5 DEAD-box RNA helicase gene encodes a novel miRNA
}

\author{
HAYLEY C. MOORE, ${ }^{1}$ MICHAEL JOHNSTON, ${ }^{2}$ SAMANTHA M. NICOL, ${ }^{1}$ JEAN-CHRISTOPHE BOURDON, ${ }^{1}$ \\ ALASTAIR M. THOMPSON, ${ }^{1}$ GYORGY HUTVAGNER, ${ }^{2,3}$ and FRANCES V. FULLER-PACE ${ }^{1}$ \\ ${ }^{1}$ Centre for Oncology \& Molecular Medicine, University of Dundee, Ninewells Hospital \& Medical School, Dundee, DD1 9SY, United Kingdom \\ ${ }^{2}$ Wellcome Trust Centre for Gene Regulation and Expression, College of Life Sciences, University of Dundee, Dundee, DD1 9SY, \\ United Kingdom \\ ${ }^{3}$ Centre for Health Technologies, Faculty of Engineering and Information Technology, University of Technology, Sydney, NSW 2007, Australia
}

\begin{abstract}
The DEAD-box RNA helicase p68 (DDX5) plays important roles in several cellular processes, including transcription, pre-mRNA processing, and microRNA (miRNA) processing. p68 expression is growth and developmentally regulated, and alterations in p68 expression and/or function have been implicated in tumor development. The p68 gene encodes an evolutionarily conserved, alternatively spliced, intron the function of which has to date remained unclear. Although the intron-containing p68 RNA does not appear to yield an alternative p68 protein, it is differentially expressed in cell lines and tissues, indicating regulation of expression. Here we show that the p68 conserved intron encodes a novel putative miRNA, suggesting a previously unknown possible regulatory function for the p68 intron. We show that this miRNA (referred to as p68 miRNA) is processed from the intron via the canonical miRNA-processing pathway and that it associates with the Argonaute protein Ago2. Finally we show that the p68 miRNA suppresses an mRNA bearing complementary target sequences, suggesting that it is functional. These findings suggest a novel mechanism by which alterations in p68 expression may impact on the cell.
\end{abstract}

Keywords: p68/DDX5; RNA helicase; alternatively spliced intron; miRNA

\section{INTRODUCTION}

p68 (DDX5) is one of the prototypic members of the DEAD-box family of RNA helicases (Ford et al. 1988), which includes a large number of proteins that participate in virtually all processes involving RNA metabolism (for review, see Linder 2006). Like several other DEAD-box proteins, p68 has been highly conserved through phylogeny with the yeast and human homologs sharing $\sim 55 \%$ identity at the amino acid level (Iggo et al. 1991), implying an important conserved function(s) for p68 in the cell. Several reports have demonstrated that p68 expression is growth and developmentally regulated (Stevenson et al. 1998; Seufert et al. 2000; Kitamura et al. 2001; Kahlina et al.

Reprint requests to: Frances V. Fuller-Pace, Centre for Oncology \& Molecular Medicine, University of Dundee, Ninewells Hospital \& Medical School, Dundee, DD1 9SY, UK; e-mail: f.v.fullerpace@dundee.ac.uk; fax: 44-1382-496361, or Gyorgy Hutvagner, Wellcome Trust Centre for Gene Regulation and Expression, College of Life Sciences, University of Dundee, Dundee, DD1 9SY, UK; e-mail: g.hutvagner@dundee.ac.uk.

Article published online ahead of print. Article and publication date are at http://www.rnajournal.org/cgi/doi/10.1261/rna.2591611.
2004), while p68 knockout in mice results in embryonic lethality at approximately embryonic day $11.5(\sim \mathrm{E} 11.5)$, underscoring the importance of this protein. Additionally, p68 is aberrantly expressed and/or post-translationally modified in a range of cancers (Causevic et al. 2001; Yang et al. 2005; Shin et al. 2007; Clark et al. 2008; Wortham et al. 2009), suggesting that changes in p68 levels and/or function may be important in cancer development.

p68 is a multifunctional protein and has been shown to be involved in multiple cellular processes, including premRNA processing (Liu 2002), alternative splicing (Guil et al. 2003; Clark et al. 2008), ribosomal RNA processing (Bond et al. 2001), nonsense-mediated decay (Bond et al. 2001), RNAi and miRNA processing (Ishizuka et al. 2002; Fukuda et al. 2007), and transcriptional regulation (for review, see Fuller-Pace 2006). As a transcriptional coregulator, p68 has been found to coactivate several transcription factors that are themselves highly regulated (e.g., Estrogen Receptor $\alpha$ [Endoh et al. 1999], the tumor suppressor p53 [Bates et al. 2005], Androgen Receptor [Clark et al. 2008], the myogenic regulator MyoD [Caretti et al. 2006], and the osteoblast differentiation factor Runx2 
[Jensen et al. 2008]). Interestingly, these studies showed that while p68 RNA helicase activity appears to be important for some functions (e.g., RNA processing), it does not appear to be required for its role as a transcriptional coactivator.

The p68 gene contains a large intron (intron $11,1.2 \mathrm{~kb}$ in the human gene), which has been conserved through evolution, being present even in yeast (Iggo et al. 1991; Rossler et al. 2000). Several studies have demonstrated the presence of two predominant p68 RNAs in cell lines and tissues, resulting from alternative splicing of this intron, and shown that their expression is differentially regulated, suggesting that the intron-containing RNA is not only stable but may also be functional (Stevenson et al. 1998; Rossler et al. 2000). Inclusion of the intron introduces a stop codon, but, to date, there is no evidence for the presence of a truncated protein. In yeast, the p68 RNA that contains this large intron can negatively regulate expression of p68 protein levels (Barta and Iggo 1995), again suggesting that this intron is functionally important. However, in mammalian tissues, there is no obvious positive, or negative, correlation between levels of intron-containing p68 RNA and p68 protein (Stevenson et al. 1998). Therefore, the function of this conserved intron has remained unclear.

miRNAs are small non-coding RNAs that post-transcriptionally regulate expression of mRNAs by base-pairing with complementary sequences, usually within the $3^{\prime}$ UTR of the targeted mRNA (Lewis et al. 2003). They are sequentially processed from primary transcripts (pri-miRNA) by the Drosha/Microprocessor complex into precursor miRNAs (pre-miRNA) (Lee et al. 2003; Han et al. 2004). Pre-miRNAs are further processed by Dicer to produce the mature miRNA duplex (Grishok et al. 2001; Hutvagner et al. 2001). One strand of the miRNA guides the RNAinduced silencing complex (RISC) to target mRNAs, resulting in inhibition of translation and/or degradation of the mRNA (for review, see Hock and Meister 2008). It has become increasingly clear that miRNAs can regulate virtually every cellular process and that their aberrant expression can contribute to the pathogenesis of many diseases, and there is growing evidence that miRNA dysregulation plays a key role in cancer development and progression (for review, see Croce 2009).

In an analysis of the sequence of the p68 intron, we found that it encodes a putative miRNA not previously annotated, suggesting a previously unknown possible regulatory function for the p68 intron. Here we show that this novel p68 intron-encoded small RNA (herein referred to as the p68 miRNA) is expressed endogenously in a range of cell lines and is processed from the p68 intron via the canonical miRNA-processing pathway. Moreover, we demonstrate that the mature p68 miRNA coimmunoprecipitates with Argonaute2 (Ago2) and that it is functional since it suppresses expression of an mRNA bearing complementary target sequences. Taken together, these findings in- dicate that the conserved p68 intron encodes a novel miRNA. In this respect, the previous observations that expression of the intron-containing RNA appears to be differentially regulated (Stevenson et al. 1998; Rossler et al. 2000) would provide an additional mechanism by which alterations in p68 expression may impact on the cell.

\section{RESULTS AND DISCUSSION}

\section{The conserved p68 intron potentially encodes a conserved miRNA}

Analysis of the sequence conservation of the p68 gene in mammals (UCSC Genome Browser) shows that the alternatively spliced intron (intron 11) of p68 is highly conserved, while, with the exception of part of intron 1, the other introns show little conservation between species (Fig. 1A). This observation, coupled with the fact that the p68 RNA, which includes this intron, is stable and differentially expressed between tissues (Stevenson et al. 1998; Rossler et al. 2000), suggests that sequences within this intron are important. However, to date, the function of this intron has remained unclear. Analysis of this intron sequence using "One-ClassMiRNAfind" (http://wotan.wistar.upenn.edu/ OneClassmiRNA) (Yousef et al. 2006) identified a putative, novel miRNA that has not previously been described in the miRBase database (Fig. 1B). Subsequent folding (RNAfold) of the RNA sequence corresponding to the predicted miRNA precursor revealed a characteristic stem-loop structure, with the predicted miRNA sequence lying on one arm of the stem (Fig. 1C). Both the predicted p68 miRNA and the primary miRNA are very highly conserved in mammals, with a high level of conservation extending to opossum and chicken and a lower level in Xenopus (Fig. 1B,D) but not to zebrafish (data not shown).

\section{The p68 intron-encoded predicted miRNA is expressed in cells and is derived from the intron}

In order to demonstrate that the predicted p68 miRNA is expressed in cell lines, total RNA isolated from triplicate samples of MCF-7 and U2OS cells were subjected to sensitive Northern hybridization (Pall et al. 2007) using a probe complementary to the predicted p68 miRNA sequence (Supplemental Table 1A). The Northern blot revealed the presence of two specific hybridizing bands with the sizes of $\sim 21 \mathrm{nt}$ and $\sim 90 \mathrm{nt}$ long, respectively, in both cell lines (Fig. 2A). The size of these fragments corresponds to the characteristic sizes of the mature and pre-miRNAs. To complement this approach, and to quantitatively analyze expression of the putative mature p68 miRNA by qRTPCR, a custom primer was designed by QIAGEN based on the predicted mature miRNA sequence (QIAGEN miScript system) to specifically amplify the mature form of the p68 intron-generated miRNA (see Materials and Methods; 
A

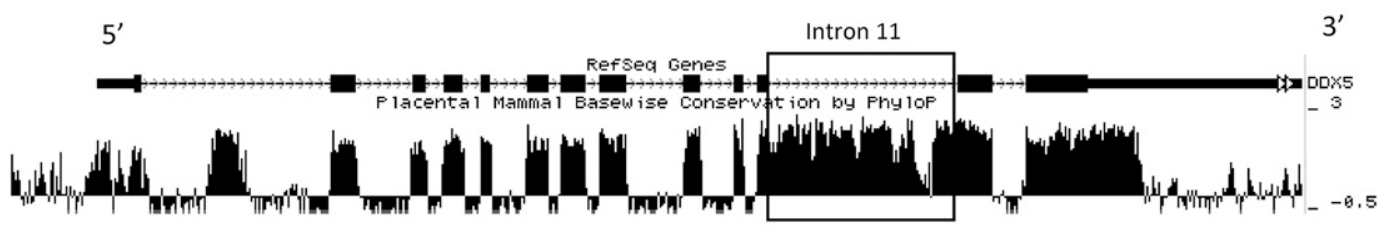

B

\begin{tabular}{|c|c|}
\hline Human & UUGCAGCUGCGGUUGUAAGGU \\
\hline Mouse & UUGCAGCUGCaGUUGUAAGGU \\
\hline Dog & UUGCAGCUGCGGUUGUAAGGU \\
\hline Opossum & UUGCAGCUGCGGUUGUAAGGU \\
\hline Chicken & UUGCAGCUGCaGUUCaAAGGU \\
\hline X. tropicalis & UUGucGCUcaacUUGUAAGGU \\
\hline
\end{tabular}

C

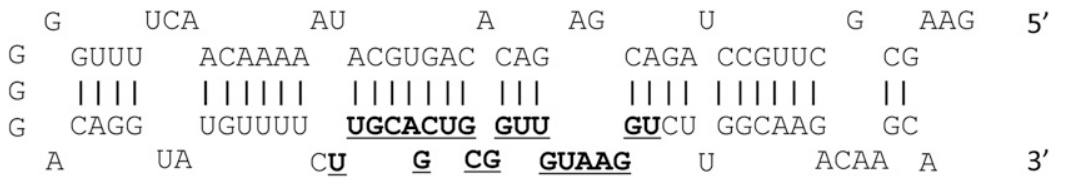

D

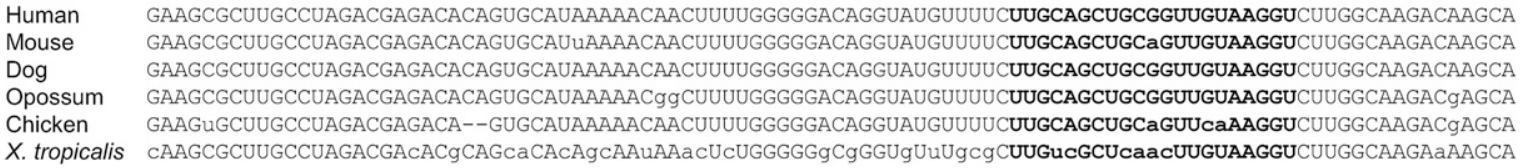

FIGURE 1. Conservation of p68 intron 11 and the putative p68 miRNA. (A) p68 gene structure and conservation as shown in the UCSC Genome Browser. The highly conserved intron 11 is labeled and highlighted with a box. The degree of conservation between mammals is shown below the gene structure with the height of the peak correlating with the extent of conservation. (B) Sequence of the predicted mature p68 miRNA and its conservation between species. $(C)$ Structure of the $\mathrm{p} 68$ pre-miRNA as predicted by RNAfold (http://rna.tbi.univie.ac.at/cgi-bin/RNAfold.cgi). The predicted mature p68 miRNA sequence is highlighted in bold and is underlined. $(D)$ Sequence of the predicted p68 pre-miRNA and its conservation between species.

Supplemental Table 1B). Similarly, primers to specifically detect RNA containing intron 11 of the p68 gene and appropriate controls were designed and purchased accordingly (Supplemental Table 1C). Using this method, we demonstrated that the putative mature p68 miRNA is expressed at relatively low levels, which are similar to those of miR-19a (a well-annotated miRNA) (Fig. 2B). Moreover, we showed that the putative p68 miRNA is differentially expressed across a range of breast cancer cell lines and that its expression correlates with the expression of the p68 intron (Fig. 2C), suggesting that it is derived from the p68 intron.

Consistent with the idea that the putative p68 miRNA is processed from the $\mathrm{p} 68$ conserved intron, overexpression of the intron in MCF-7 cells resulted in increased levels of the p68 miRNA (Fig. 2D), while an siRNA targeted against sequences within the intron, but outside the region corresponding to the p68 miRNA, resulted in a decrease in p68 miRNA expression (Fig. 2E). Similar results were obtained with U2OS cells (data not shown). (siRNA sequences are shown in Supplemental Table 1D.) Moreover, overexpression or siRNA knockdown of the p68 intron did not result in significant alterations of the levels of U1 small nuclear RNA (RNU1A) or p68 mRNA, ruling out non-specific effects of intron overexpression/siRNA knockdown (data not shown). These complementary approaches confirm that the putative p68 miRNA is indeed derived from the p68 intron.

\section{The p68 intron-encoded small RNA is a bona fide miRNA}

It is well established that decreased levels of the key proteins of the miRNA processing and maturation pathway result in an attenuation of the steady-state level of miRNAs (for review, see Kim et al. 2009). Therefore, in order to demonstrate that the small RNA derived from the p68 intron is indeed a bona fide miRNA that is processed via the canonical miRNA-processing pathway, we knocked down the proteins of this processing pathway by siRNA (Supplemental Table 1D) and monitored the effects of such knockdowns on the expression of the p68 intron-encoded miRNA. Firstly, we targeted the Microprocessor complex 


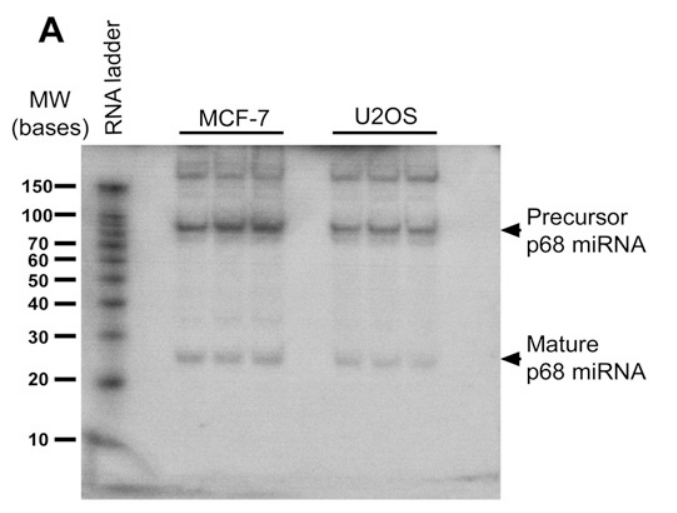

B
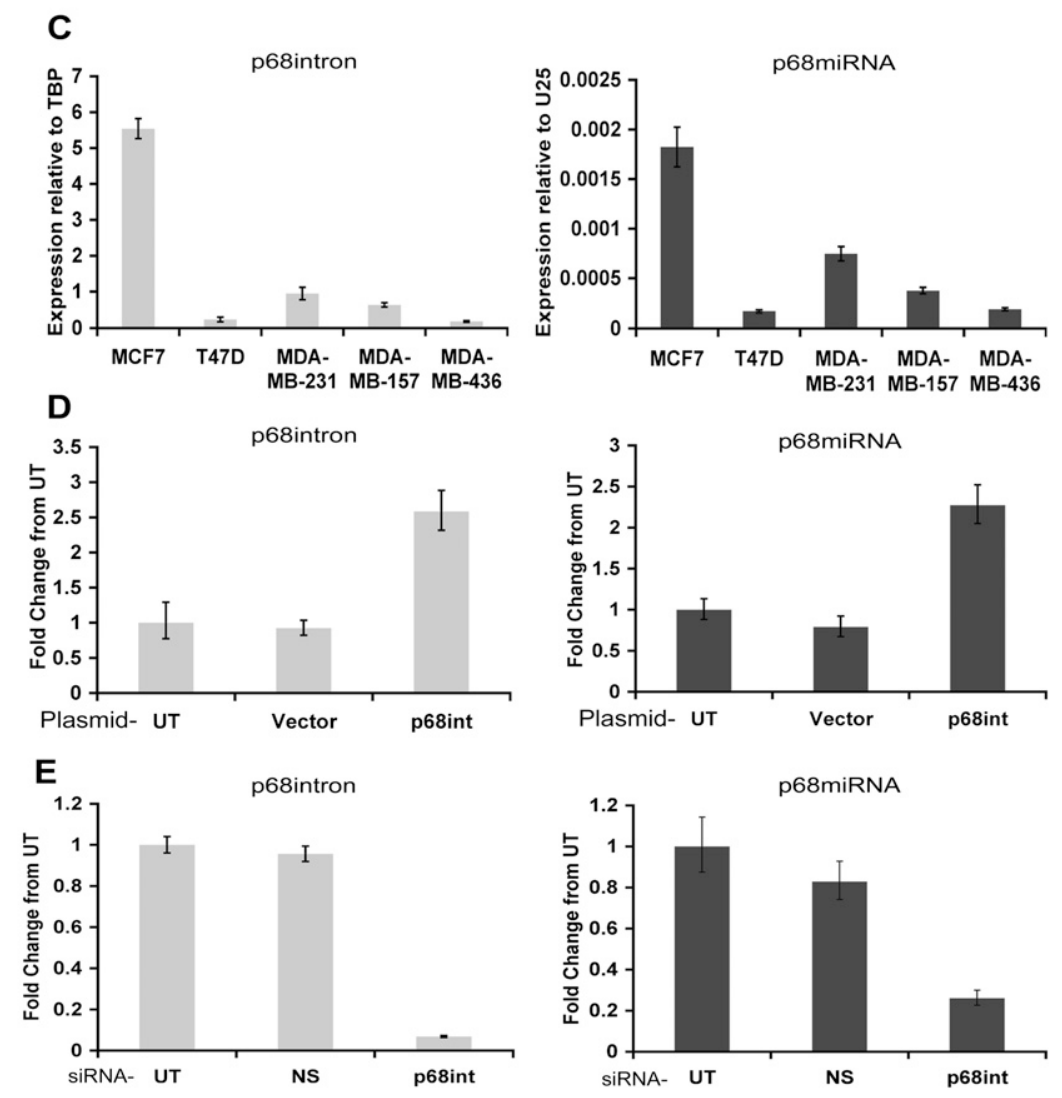

FIGURE 2. Expression of the predicted p68 miRNA and intron 11-containing p68 RNA. $(A)$ Northern blot to detect the predicted p68 miRNA in MCF-7 and U2OS cells using a probe complementary to the predicted sequence. RNA species corresponding to the size of the mature p68 miRNA and the pre-p68 miRNA were detected. (B) qRT-PCR demonstrating expression of the p68 miRNA in MCF-7 cells as compared with other well-annotated miRNAs. (C) qRT-PCR showing expression of p68 intron 11-containing RNA and the mature p68 miRNA in a range of cell lines. $(D)$ qRT-PCR showing expression of p68 intron 11-containing RNA and the p68 miRNA in MCF-7 cells transfected with a plasmid encoding the p68 intron 11 or an empty vector control. (E) qRT-PCR showing expression of p68 intron 11-containing RNA and the p68 miRNA in MCF-7 cells transfected with an siRNA targeting the p68 intron or a non-specific siRNA. In graphs $B$ and $C$ expression of intron 11-containing p68 RNA (p68 intron) and p68 miRNA was calculated relative to TBP (TATA binding protein) and U25 (SNORD25), respectively. In graphs $D$ and $E$ values are calculated as fold change from untransfected cells. In all graphs the average values from three independent experiments are shown \pm SEM. (UT) Untransfected cells; (NS) non-specific; (p68int) p68 intron 11. with an siRNA against Drosha, which is involved in processing of the primary miRNA transcript into the precursor miRNA. This resulted in a decrease in the levels of mature putative p68 miRNA, as measured by qRT-PCR (Fig. 3A). Consistent with this finding, an accumulation of the candidate primary p68 miRNA transcript (the p68 intron) was observed (Fig. 3B). Secondly, we knocked down Dicer, which processes the pre-miRNA into a doublestranded miRNA intermediate. The impairment of Dicer expression again resulted in a reduction in the putative p68 miRNA (Fig. 3C). Thirdly, we knocked down the Argonaute protein Ago2, which plays an active role in the maturation of miRNAs by facilitating the generation of single-stranded mature miRNAs from the double-stranded intermediate duplexes (Matranga et al. 2005). This again resulted in a decrease in the levels of the putative p68 miRNA (Fig. 3D). In all cases, a non-specific siRNA was used as a control. As additional controls, the levels of miR-19a and its primary transcript miR-17-92 were monitored. This showed that miR19a was similarly reduced by Drosha, Dicer, or Ago2 knockdown, while the miR-17-92 transcript was increased by Drosha knockdown, confirming that the putative p68 miRNA is processed in a similar way to miR-19a. For each experiment Western blotting was used to monitor the efficiency of the Drosha, Dicer, and Ago2 knockdowns (Fig. 3E), and specific effects of the siRNAs on general RNA processing were ruled out by showing that there were no effects on RNU1A expression (data not shown).

\section{The p68 miRNA is a functional miRNA}

miRNAs that are involved in translational repression or target cleavage are found bound to an effector Argonaute protein, forming the minimal RISC (for review, see Hutvagner and Simard 2008). To determine whether the putative p68 miRNA is functional, we immunoprecipitated Ago2 from MCF-7 cells and carried out miRNA-specific 
qRT-PCR to detect coimmunoprecipitating miRNAs. Using this approach, we showed that the p68 miRNA is present in the Ago2 immunoprecipitate (IP) at a level comparable to that of miR-19a (Fig. 3F), which is expressed at a similar level (Fig. 2B). This finding suggests that the p68 miRNA is efficiently incorporated into Ago2 RISC complexes. A Flag IP was used as a control to rule out non-specific precipitation of the p68 miRNA; this was further confirmed by Western blotting of the immunoprecipitates (Fig. 3G).

Endogenous miRNAs can mediate sequence-specific RNA cleavage or repress translation of specific mRNAs if a target with perfect complementarity is presented. Therefore, to demonstrate that the p68 miRNA is functional, a luciferase biosensor assay, using the psiCHECK-2 reporter system, was designed in which the Renilla luciferase is regulated by an artificial 3' UTR containing three copies of the complementary target site of the p68 miRNA (Supplemental Table 1E). As a control, we generated a similar sensor reporter in which we mutated a number of bases in the p68 miRNA complementary sequences to prevent sequence-specific cleavage or translational repression possibly mediated by the p68 miRNA (Supplemental Table 1E). When these reporter plasmids were transfected into MCF-7 cells, the expression level of the p68 miRNA-targeted reporter was significantly lower than that of the reporter with the mutated p68 miRNA target sites (Fig. $3 \mathrm{H}$ ), suggesting that the p68 miRNA recognizes and regulates expression through the complementary sequences built in the miRNA sensor. This effect was slightly, but significantly ( $p=0.05)$, more prominent when the p68 intron was co-transfected with the sensor reporter plasmids. These findings are consistent with the observation that higher expression levels of the intron result in increased steady-state level of p68 miRNA (Fig. 2D), which may, in turn, enhance the repression of the p68 miRNA sensor reporter (Fig. $3 \mathrm{H}$ ). To test that the effect of the overexpression of the p68 miRNA-expressing p68 intron is specific to the regulation of the p68 miRNA sensor, we co-transfected the p68 intron with a sensor that is

A

$\mathrm{E}$

F
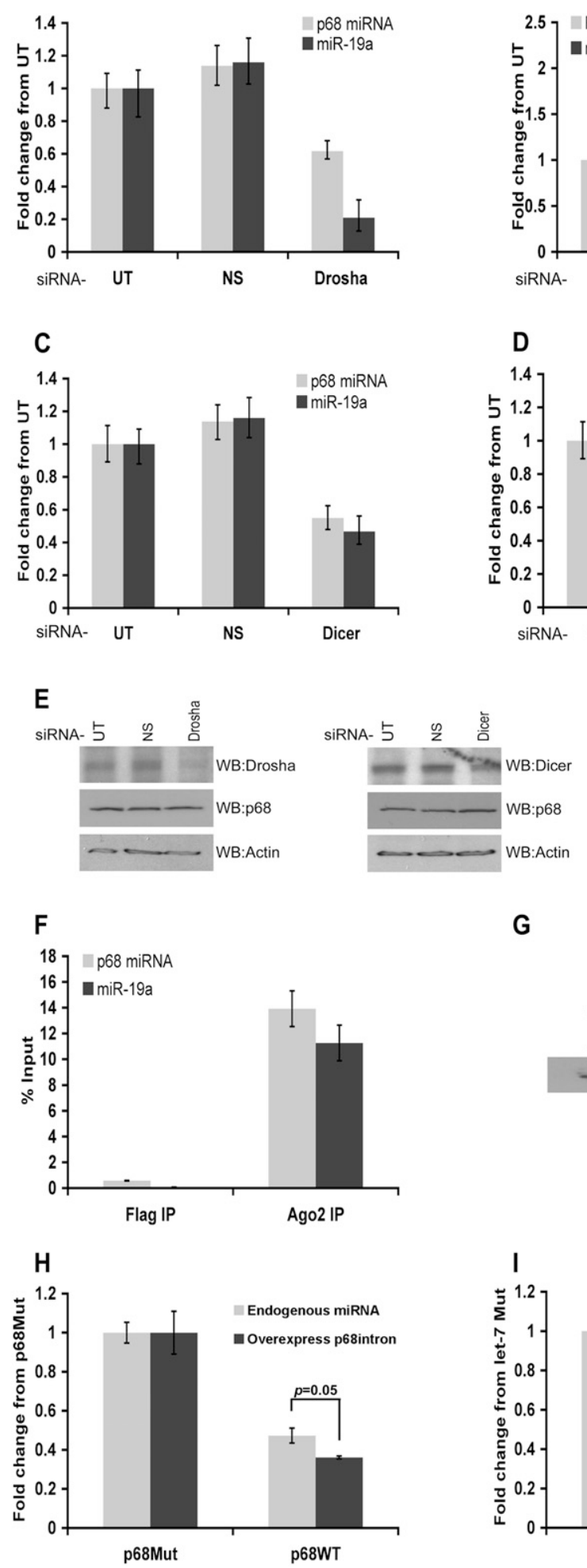

FIGURE 3. (Legend on next page)

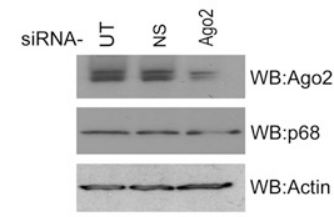

G
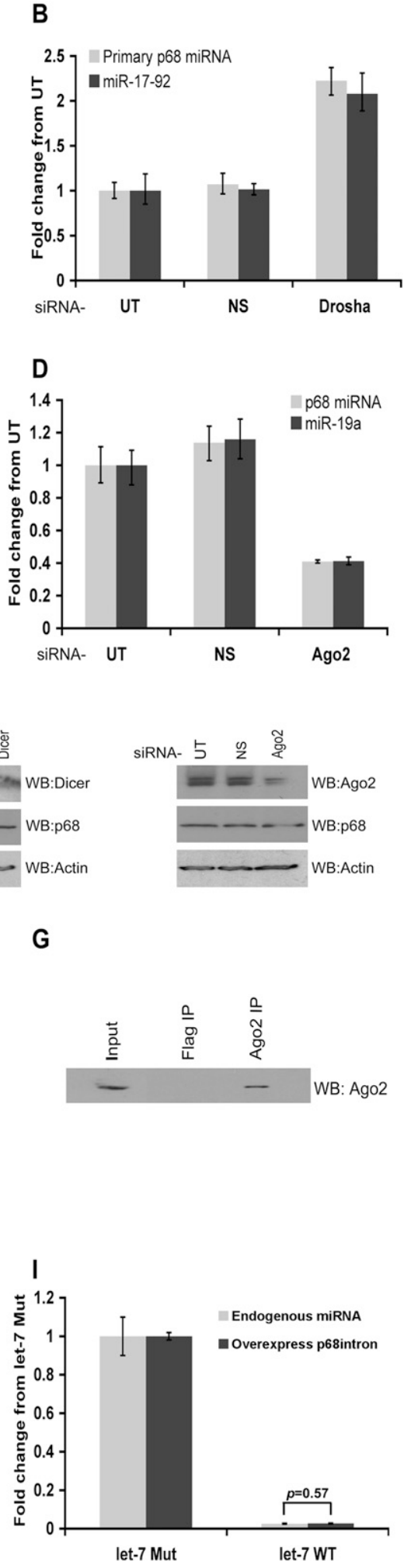

tet-7 Mut

let-7 WT 
expression of the let-7 sensor, suggesting that the regulation mediated by the p68 intron-generated miRNA is specific (Fig. 3I). It should be noted that the levels of expression of the sensor including let-7 complementary sequences are very low compared with those of the mutated let-7 miRNA target site, possibly reflecting the higher abundance of endogenous let-7 miRNA. Nevertheless, no enhanced repression of the let-7 miRNA was observed following overexpression of the p68 intron $(p=0.57)$ (Fig. $3 \mathrm{I})$ as compared with that seen with the p68 miRNA sensor (Fig. $3 \mathrm{H}$ ).

In summary, we have identified a novel, not previously annotated, miRNA encoded within a large conserved intron (intron 11) of the p68 gene. The sequence of the p68 miRNA is well conserved among species, consistent with the idea that many miRNAs are evolutionarily conserved with possible conserved functions (for review, see Niwa and Slack 2007). Moreover, the expression of the p68 miRNA correlates with that of the p68 conserved intron, and its expression can be modulated by either overexpressing or knocking down of the intron, suggesting that the p68 miRNA is indeed generated from this intron. We have shown that the p68 miRNA is a bona fide miRNA since it is generated through the canonical miRNA processing pathway and that the mature p68 miRNA is able to form a complex with human Ago2. Furthermore, the p68 miRNA is able to specifically repress the expression of a luciferase reporter plasmid containing complementary target sequences in its $3^{\prime}$ UTR, strongly suggesting that the p68 miRNA is functional and has the potential to regulate endogenous targets.

More than 2000 miRNAs have been described to date, with many more miRNAs being discovered due to improvements in prediction software and the extensive use of massive parallel sequencing technology. However, this is the first report of a miRNA encoded within the p68 gene. It is very interesting that extensive new-generation sequencing of small RNA libraries has not yet revealed the p68derived miRNA despite the fact that deep sequencing data are available for U2OS (http://www.ncbi.nlm.nih.gov/geo/ query/acc.cgi? acc=GSM416754) and MCF-7 (http://www. ncbi.nlm.nih.gov/geo/query/acc.cgi?acc=GSM416760) cells. We have searched this database but did not find sequences that are similar to the p68 miRNA. In contrast, miR-19a, which is expressed at a level comparable to that of the p68 miRNA, was represented in both libraries with low frequency compared with the most abundant miRNAs (2803 reads in U2OS and 261 in MCF-7). The simplest explanation for this discrepancy is that the p68 miRNA is difficult to clone using recent technology and is therefore under-represented in deep sequencing libraries. This possibility is supported by the fact that we failed to identify the $5^{\prime}$-end of the mature p68 miRNA using a 5'-RACE approach (data not shown). Indeed, it has recently been shown that deep sequencing of small RNA libraries is biased and the library-making process generates this bias (Linsen et al. 2009). The majority of mammalian miRNAs are encoded within introns of transcriptional units and are transcribed by RNA polymerase II as part of the host gene (Rodriguez et al. 2004; Kim and Kim 2007; Rearick et al. 2010). The presence of the p68 miRNA within intron 11 of the p68 gene may explain the high evolutionary conservation of this intron and suggests a conserved function. Investigations aimed at understanding the regulation of the p68 miRNA, as well as the identification of endogenous targets, are required to provide a clearer picture of the role of this novel miRNA, and of the p68 intron 11-containing RNA from which it is derived.
FIGURE 3. The intron 11-encoded small RNA is a bona fide miRNA. MCF-7 cells were transfected with an siRNA targeted against Drosha and expression of $(A)$ p68 miRNA and miR-19a; or $(B)$ the primary p68 miRNA transcript (intron 11 ) and the miR-17-92 cluster (primary sequence of miR-19a) were determined by qRT-PCR. Expression of p68 miRNA and miR-19a in MCF-7 cells treated with an siRNA-targeting $(C)$ Dicer or $(D)$ Ago2 was measured similarly. Expression levels for $A, B$, and $C$ were calculated relative to U25 and relative to TBP for $B$, and expressed as fold change from untransfected cells (UT). (E) Corresponding Western blots showing knockdown of Drosha, Dicer, and Ago2. Actin was used as a loading control, and expression of p68 was examined to rule out any non-specific effects. $(F)$ Immunoprecipitation (IP) of p68 miRNA with endogenous Ago2 in MCF-7 cells. A Flag IP was also carried out as a negative control. The amount of p68 miRNA and miR-19a (as control) immunoprecipitated by Ago2 was quantified by qRT-PCR and calculated as percentage of input. $(G)$ Corresponding Western blot of Ago2 and Flag immunoprecipitants. A luciferase biosensor assay was designed in which three copies of $(H)$ the p68 miRNA (p68 WT) or $(I)$ let-7 (let-7 WT) target sites were cloned downstream from the Renilla luciferase gene in the psiCHECK-2 dual luciferase vector. As controls, mutations were made in the target sites (p68 Mut and let-7 Mut). MCF-7 cells were transfected with these reporter constructs, and Renilla luciferase activity was measured for both endogenous miRNA expression and also after overexpression of p68 intron 11. Renilla luciferase activity was calculated relative to firefly luciferase and plotted as fold change from the plasmid containing the mutated target sites. A Student's $t$-test was used to determine significance of the effects of overexpression of p68 intron 11 on expression of the wild-type (WT) p68 miRNA $(H)$ or the let-7 target $(I)$. In all graphs the average values from three independent experiments are shown \pm SEM. (UT) Untransfected; (NS) non-specific; (WB) Western blot.

\section{MATERIALS AND METHODS}

\section{Cell lines}

Cell lines used in this study include the breast carcinoma MCF-7, T47D, MDA-MB157, MDA-MB-231, MDA-MB-436, and the osteosarcoma U2OS. All cell lines were maintained in Dulbecco's Modified Eagle's Medium (DMEM) supplemented with 10\% fetal calf serum (FCS), 2 mM L-glutamine, $100 \mu \mathrm{g} / \mathrm{mL}$ streptomycin, and $100 \mathrm{U} / \mathrm{mL}$ penicillin (all supplied by Invitrogen) in 5\% $\mathrm{CO}_{2}$ at $37^{\circ} \mathrm{C}$.

\section{Plasmids}

The p68 intron was cloned into pcDNA3.1(+) for transient transfections. Three copies of the antisense sequence to the p68 miRNA or let-7 (or mutated versions) were cloned in the 
psiCHECK-2 dual luciferase vector (Promega) to provide targets in the 3' UTR. Sequences are shown in Supplemental Table 1E for the p68 miRNA and in Johnston et al. (2010) for let-7.

\section{Antibodies}

The following antibodies were used: p68-PAb204 (mouse monoclonal; Millipore); Dicer-ab14601 (mouse monoclonal; Abcam); Drosha-07-717 (rabbit polyclonal; Millipore); Ago2-11A9 (mouse monoclonal; a gift from Gunter Meister; Rudel et al. 2008); and actin-A2066 (rabbit polyclonal; Sigma). Appropriate HRP-conjugated anti-mouse, anti-rabbit, and anti-rat secondary antibodies for immunoblotting were purchased from DAKO.

\section{Plasmid DNA and siRNA transfections}

Plasmids expressing the p68 intron under the control of a CMV promoter were transfected using Fugene6 (Roche) according to the manufacturer's protocol. siRNA reverse transfections were carried out using Lipofectamine RNAiMax (Invitrogen) in MCF-7 cells or Lipofectamine 2000 (Invitrogen) in U2OS cells and 100 pmol of siRNA against Drosha, Dicer, Ago2 (see Supplemental Table 1), p68 intron 11 (Dharmacon), or a non-silencing control (Dharmacon) according to the manufacturers' instructions (Supplemental Table 1D).

\section{Luciferase assays}

MCF-7 cells were transfected using Fugene6 (Roche) with luciferase reporters as indicated in figure legends. Luciferase activity was measured $24 \mathrm{~h}$ after transfection using the Dual-Glo luciferase kit (Promega) (Johnston et al. 2010).

\section{RNA extraction and quantitative RT-PCR (qRT-PCR)}

Total RNA including small RNA was extracted from cells using the miRNeasy kit (QIAGEN) according to the manufacturer's protocol. Two micrograms of total RNA was reverse-transcribed using the miScript Reverse Transcription Kit (QIAGEN). Using this system, miRNAs are polyadenylated by poly(A) polymerase during the reverse-transcription step. All polyadenylated RNAs are converted to cDNA using oligo $(\mathrm{dT})$ and random primers. The oligo(dT) primer contains a unique tag sequence on the $5^{\prime}$-end, which is later used to amplify the miRNA during the PCR step.

Quantitative PCR was carried out using the Stratagene MX3005-P instrument. The mature p68 miRNA was detected by PCR using a forward primer assay specific to this miRNA (derived from the predicted sequence and designed by QIAGEN) and a reverse universal primer complementary to the tag sequence added during the reverse-transcription step. Primer assays were purchased from QIAGEN for all other miRNA and small RNAs. Furthermore, the cDNA generated could also be used to detect longer RNAs (e.g., p68 intron-containing RNA) using gene-specific primers designed using Primer Express Version 1.0 software (Applied Biosystems) and purchased from MWG. Primer assay IDs for miRNAs/small RNAs and sequences of the gene-specific primers used are shown in Supplemental Tables 1B and 1C.

Quantification of miRNA and mRNA (including the p68 intron 11-containing RNA) was carried out using the miScript SYBR Green PCR kit (QIAGEN) and the QuantiFast SYBR Green PCR Mix (QIAGEN), respectively. SNORD25 (U25) and TBP were used as controls for quantification of miRNA and mRNA, respectively. Fold changes and differences between samples were calculated using the $\Delta \Delta \mathrm{Ct}$ method in Microsoft Excel.

\section{Northern blot}

Total RNA was prepared using Trizol (Invitrogen) according to the manufacturer's protocol. Five micrograms of total RNA derived from each sample was separated on a $15 \%$ denaturing polyacrylamide gel. Specialized Northern blot conditions, as described by Pall et al. (2007), were used to detect miRNAs by crosslinking RNA to the membrane using EDC (1-ethyl-3-[3-dimethylaminopropyl] carbodiimide; Sigma). The oligonucleotide probe used to detect the p68 miRNA was complementary to the predicted p68 miRNA sequence and end-labeled using $\left[\gamma^{32} \mathrm{P}\right]$ (sequence shown in Supplemental Table 1A).

\section{Argonaute 2 (Ago2) immunoprecipitation}

MCF-7 cells were washed twice in warm $1 \times$ PBS and once in cold $1 \times$ PBS, followed by lysis in NP-40 lysis buffer $(50 \mathrm{mM}$ Tris- $\mathrm{HCl}$ at $\mathrm{pH}$ 7.5, $150 \mathrm{mM} \mathrm{NaCl}, 1 \%$ [v/v] NP-40, $10 \mu \mathrm{M}$ DTT, 40 units/ $\mathrm{mL}$ RNase inhibitor [NEB], and one complete protease inhibitor tablet [Roche] per $10 \mathrm{~mL}$ of buffer). Immunoprecipitation of Ago 2 was performed using the rat monoclonal anti-Ago 2 antibody (11A9) (Rudel et al. 2008) as described previously (Johnston et al. 2010). Ten microliters of each IP was removed for analysis by Western blot, and the remaining $40 \mu \mathrm{L}$ was used for RNA isolation, using phenol/chloroform, to detect Ago2-associated RNA.

\section{SUPPLEMENTAL MATERIAL}

Supplemental material is available for this article.

\section{ACKNOWLEDGMENTS}

This work was supported by grants from the Breast Cancer Campaign, Tenovus Scotland, Cancer Research UK, the Medical Research Council, and the Wellcome Trust. We thank Malik Yousef for help with the miRNA fold analysis and Gunter Meister for the Ago2 antibody.

\section{NOTE ADDED IN PROOF}

The p68 miRNA sequence has been deposited in the "miRBase" microRNA database and has been assigned the number hsa-mir5047.

Received December 14, 2010; accepted January 28, 2011.

\section{REFERENCES}

Barta I, Iggo R. 1995. Autoregulation of expression of the yeast Dbp2p 'DEAD-box' protein is mediated by sequences in the conserved DBP2 intron. EMBO J 14: 3800-3808.

Bates GJ, Nicol SM, Wilson BJ, Jacobs AM, Bourdon JC, Wardrop J, Gregory DJ, Lane DP, Perkins ND, Fuller-Pace FV. 2005. The DEAD box protein p68: a novel transcriptional coactivator of the p53 tumour suppressor. EMBO J 24: 543-553. 
Bond AT, Mangus DA, He F, Jacobson A. 2001. Absence of Dbp2p alters both nonsense-mediated mRNA decay and rRNA processing. Mol Cell Biol 21: 7366-7379.

Caretti G, Schiltz RL, Dilworth FJ, Di Padova M, Zhao P, Ogryzko V, Fuller-Pace FV, Hoffman EP, Tapscott SJ, Sartorelli V. 2006. The RNA helicases p68/p72 and the noncoding RNA SRA are coregulators of MyoD and skeletal muscle differentiation. Dev Cell 11: 547-560.

Causevic M, Hislop RG, Kernohan NM, Carey FA, Kay RA, Steele RJ, Fuller-Pace FV. 2001. Overexpression and poly-ubiquitylation of the DEAD-box RNA helicase p68 in colorectal tumours. Oncogene 20: 7734-7743.

Clark EL, Coulson A, Dalgliesh C, Rajan P, Nicol SM, Fleming S, Heer R, Gaughan L, Leung HY, Elliott DJ, et al. 2008. The RNA helicase p68 is a novel androgen receptor coactivator involved in splicing and is overexpressed in prostate cancer. Cancer Res 68: 7938-7946.

Croce CM. 2009. Causes and consequences of microRNA dysregulation in cancer. Nat Rev Genet 10: 704-714.

Endoh H, Maruyama K, Masuhiro Y, Kobayashi Y, Goto M, Tai H, Yanagisawa J, Metzger D, Hashimoto S, Kato S. 1999. Purification and identification of p68 RNA helicase acting as a transcriptional coactivator specific for the activation function 1 of human estrogen receptor $\alpha$. Mol Cell Biol 19: 5363-5372.

Ford MJ, Anton IA, Lane DP. 1988. Nuclear protein with sequence homology to translation initiation factor eIF-4A. Nature 332: 736-738.

Fukuda T, Yamagata K, Fujiyama S, Matsumoto T, Koshida I, Yoshimura K, Mihara M, Naitou M, Endoh H, Nakamura T, et al. 2007. DEAD-box RNA helicase subunits of the Drosha complex are required for processing of rRNA and a subset of microRNAs. Nat Cell Biol 9: 604-611.

Fuller-Pace FV. 2006. DExD/H box RNA helicases: multifunctional proteins with important roles in transcriptional regulation. Nucleic Acids Res 34: 4206-4215.

Grishok A, Pasquinelli AE, Conte D, Li N, Parrish S, Ha I, Baillie DL, Fire A, Ruvkun G, Mello CC. 2001. Genes and mechanisms related to RNA interference regulate expression of the small temporal RNAs that control C. elegans developmental timing. Cell 106: 23-34.

Guil S, Gattoni R, Carrascal M, Abian J, Stevenin J, Bach-Elias M. 2003. Roles of hnRNP A1, SR proteins, and p68 helicase in c-H-ras alternative splicing regulation. Mol Cell Biol 23: 2927-2941.

Han J, Lee Y, Yeom KH, Kim YK, Jin H, Kim VN. 2004. The DroshaDGCR8 complex in primary microRNA processing. Genes Dev 18: 3016-3027.

Hock J, Meister G. 2008. The Argonaute protein family. Genome Biol 9: 210. doi: 10.1186/gb-2008-9-2-210.

Hutvagner G, Simard MJ. 2008. Argonaute proteins: key players in RNA silencing. Nat Rev Mol Cell Biol 9: 22-32.

Hutvagner G, McLachlan J, Pasquinelli AE, Balint E, Tuschl T, Zamore PD. 2001. A cellular function for the RNA-interference enzyme Dicer in the maturation of the let-7 small temporal RNA. Science 293: 834-838.

Iggo RD, Jamieson DJ, MacNeill SA, Southgate J, McPheat J, Lane DP. 1991. p68 RNA helicase: Identification of a nucleolar form and cloning of related genes containing a conserved intron in yeasts. Mol Cell Biol 11: 1326-1333.

Ishizuka A, Siomi MC, Siomi H. 2002. A Drosophila fragile X protein interacts with components of RNAi and ribosomal proteins. Genes Dev 16: 2497-2508.

Jensen ED, Niu L, Caretti G, Nicol SM, Teplyuk N, Stein GS, Sartorelli V, van Wijnen AJ, Fuller-Pace FV, Westendorf JJ. 2008. p68 (Ddx5) interacts with Runx2 and regulates osteoblast differentiation. J Cell Biochem 103: 1438-1451.

Johnston M, Geoffroy MC, Sobala A, Hay R, Hutvagner G. 2010. HSP90 protein stabilizes unloaded argonaute complexes and microscopic P-bodies in human cells. Mol Biol Cell 21: 1462-1469.

Kahlina K, Goren I, Pfeilschifter J, Frank S. 2004. p68 DEAD box RNA helicase expression in keratinocytes. Regulation, nucleolar locali- zation, and functional connection to proliferation and vascular endothelial growth factor gene expression. J Biol Chem 279: 44872-44882.

Kim YK, Kim VN. 2007. Processing of intronic microRNAs. EMBO J 26: $775-783$.

Kim VN, Han J, Siomi MC. 2009. Biogenesis of small RNAs in animals. Nat Rev Mol Cell Biol 10: 126-139.

Kitamura A, Nishizuka M, Tominaga K, Tsuchiya T, Nishihara T, Imagawa M. 2001. Expression of p68 RNA helicase is closely related to the early stage of adipocyte differentiation of mouse 3T3-L1 cells. Biochem Biophys Res Commun 287: 435-439.

Lee Y, Ahn C, Han J, Choi H, Kim J, Yim J, Lee J, Provost P, Radmark O, Kim S, et al. 2003. The nuclear RNase III Drosha initiates microRNA processing. Nature 425: 415-419.

Lewis BP, Shih IH, Jones-Rhoades MW, Bartel DP, Burge CB. 2003. Prediction of mammalian microRNA targets. Cell 115: 787-798.

Linder P. 2006. DEAD-box proteins: a family affair-active and passive players in RNP-remodeling. Nucleic Acids Res 34: 4168-4180.

Linsen SE, de Wit E, Janssens G, Heater S, Chapman L, Parkin RK, Fritz B, Wyman SK, de Bruijn E, Voest EE, et al. 2009. Limitations and possibilities of small RNA digital gene expression profiling. Nat Methods 6: 474-476.

Liu ZR. 2002. p68 RNA helicase is an essential human splicing factor that acts at the U1 snRNA-5' splice site duplex. Mol Cell Biol 22: 5443-5450.

Matranga C, Tomari Y, Shin C, Bartel DP, Zamore PD. 2005. Passenger-strand cleavage facilitates assembly of siRNA into Ago2-containing RNAi enzyme complexes. Cell 123: 607-620.

Niwa R, Slack FJ. 2007. The evolution of animal microRNA function. Curr Opin Genet Dev 17: 145-150.

Pall GS, Codony-Servat C, Byrne J, Ritchie L, Hamilton A. 2007. Carbodiimide-mediated cross-linking of RNA to nylon membranes improves the detection of siRNA, miRNA and piRNA by Northern blot. Nucleic Acids Res 35: e60. doi: 10.1093/nar/gkm112.

Rearick D, Prakash A, McSweeny A, Shepard SS, Fedorova L, Fedorov A. 2010. Critical association of ncRNA with introns. Nucleic Acids Res doi: 10.1093/nar/gkq1080.

Rodriguez A, Griffiths-Jones S, Ashurst JL, Bradley A. 2004. Identification of mammalian microRNA host genes and transcription units. Genome Res 14: 1902-1910.

Rossler OG, Hloch P, Schutz N, Weitzenegger T, Stahl H. 2000. Structure and expression of the human p68 RNA helicase gene. Nucleic Acids Res 28: 932-939.

Rudel S, Flatley A, Weinmann L, Kremmer E, Meister G. 2008. A multifunctional human Argonaute2-specific monoclonal antibody. RNA 14: 1244-1253.

Seufert DW, Kos R, Erickson CA, Swalla BJ. 2000. p68, a DEAD-box RNA helicase, is expressed in chordate embryo neural and mesodermal tissues. J Exp Zool 288: 193-204.

Shin S, Rossow KL, Grande JP, Janknecht R. 2007. Involvement of RNA helicases p68 and p72 in colon cancer. Cancer Res 67: 75727578.

Stevenson RJ, Hamilton SJ, MacCallum DE, Hall PA, Fuller-Pace FV. 1998. Expression of the 'DEAD box' RNA helicase p68 is developmentally and growth regulated and correlates with organ differentiation/maturation in the fetus. J Pathol 184: 351-359.

Wortham NC, Ahamed E, Nicol SM, Thomas RS, Periyasamy M, Jiang J, Ochocka AM, Shousha S, Huson L, Bray SE, et al. 2009. The DEAD-box protein p72 regulates ER $\alpha$-/oestrogen-dependent transcription and cell growth, and is associated with improved survival in ER $\alpha$-positive breast cancer. Oncogene 28: 4053-4064.

Yang L, Lin C, Liu ZR. 2005. Phosphorylations of DEAD box p68 RNA helicase are associated with cancer development and cell proliferation. Mol Cancer Res 3: 355-363.

Yousef M, Nebozhyn M, Shatkay H, Kanterakis S, Showe LC, Showe MK. 2006. Combining multi-species genomic data for microRNA identification using a Naive Bayes classifier. Bioinformatics 22: $1325-1334$. 

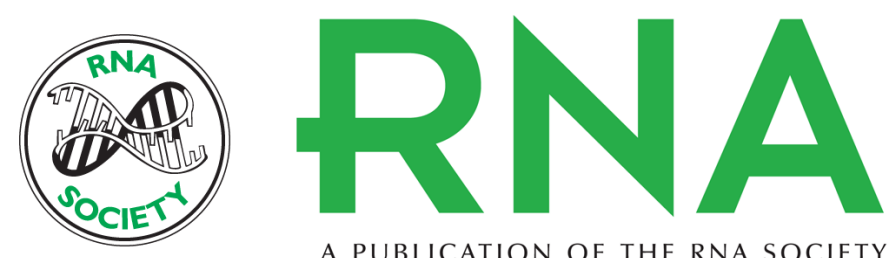

A PUBLICATION OF THE RNA SOCIETY

\section{An evolutionarily conserved, alternatively spliced, intron in the p68/DDX5 DEAD-box RNA helicase gene encodes a novel miRNA}

Hayley C. Moore, Michael Johnston, Samantha M. Nicol, et al.

RNA 2011 17: 555-562 originally published online February 23, 2011

Access the most recent version at doi:10.1261/rna.2591611

Supplemental
Material http://rnajournal.cshlp.org/content/suppl/2011/02/08/rna.2591611.DC1

References This article cites 42 articles, 15 of which can be accessed free at:

http://rnajournal.cshlp.org/content/17/4/555.full.html\#ref-list-1

License

Email Alerting Receive free email alerts when new articles cite this article - sign up in the box at the Service top right corner of the article or click here.

To subscribe to $R N A$ go to:

http://rnajournal.cshlp.org/subscriptions 